\title{
¿Cocreación
}

o participación?

Estudio de caso

de las secciones

"El periodista soy yo"

de Noticias Caracol

y "El cazanoticias"

de Noticias RCN ${ }^{1,2}$

\section{Co-creation or participation?}

Case study of the segments "El periodista

soy yo" from Caracol News and

"El cazanoticias" from RCN News

DOI: http://dx.doi.org/10.18566/comunica.n41.a05

Fecha de recepción: 1. ${ }^{\circ}$ de agosto de 2019

Fecha de aceptación: 1. de noviembre de 2019

\section{Resumen}

La industria audiovisual se está transformando: las audiencias se empoderan cada vez más de los procesos de producción y se muestran como actores importantes en los procesos de consumo y producción audiovisual. Los medios han comenzado a desarrollar propuestas para vincular al ahora llamado prosumidor. En este texto se analizan dos secciones de los informativos colombianos Noticias Caracol y Noticias RCN ("El periodista soy yo" y "El cazanoticias", respectivamente) con el fin de determinar si los procesos que desarrollan en estas con los televidentes corresponden a cocreación o participación. El análisis se centró en tres ejes fundamentales: la pieza audiovisual transmitida, los aportes del televidente y los aportes del noticiero a dicha construcción. 
Estos ejes estuvieron compuestos por variables construidas con base en diferentes datos y aportes teóricos. A partir de este análisis se pudieron identificar los niveles de participación que tanto el televidente como el noticiero tuvieron en la elaboración de la nota periodística. Como resultado, quedó evidencia de las potencialidades creativas de las audiencias y de las oportunidades y desafíos que deben afrontar los noticieros para desarrollar procesos de creación con los prosumidores.

\section{Abstract}

The audiovisual industry is changing. Audiences are empowering, appearing as agents of audiovisual production and consumption processes. Media have begun to develop alternatives to engage the now-called prosumer. In this paper, I will analyze two segments of the colombian news networks Caracol News and RCN News, in order to determine if the processes they develop with viewers in their sections "El periodista soy yo" and "El cazanoticias", respectively, correspond to processes of co-creation or participation. The analysis focused on three fundamental axes: the broadcasted audiovisual piece, the viewer's contributions and the news's contributions to this construction. These axes were composed by variables constructed from different data and theoretical contributions. From this analysis it was possible to identify participation levels that both, the viewer and the newscast had in the construction of the journalistic note. The result shows some evidence of the creative potentialities of audience, as well as opportunities and challenges related to communication that media should face in order to develop co-creation processes with prosumers.

\section{Introducción}

La industria audiovisual colombiana atraviesa cambios importantes. Los avances tecnológicos y el nuevo rol de los espectadores son los ejes principales de estas transformaciones. Los televidentes son actualmente un miembro activo dentro de todos los procesos de producción audiovisual, y no solo por el empoderamiento en los procesos de consumo, sino también por los aportes que hacen en las etapas de creación, desafiando las metodologías y técnicas habituales de producción y distribución.

Este empoderamiento de los espectadores y su incursión en diferentes etapas de la creación audiovisual han llevado a replantear algunos preceptos de la industria, como el de distribución, el pago por consumo y el derecho de autor, discusiones que se comienzan a abordar en escenarios académicos, empresariales y políticos. Estos cambios han llevado, entre otras situaciones, a que los productores audiovisuales vinculen a las audiencias en la creación audiovisual. Life in a day (Scott \& Macdonald, 2011) y El cosmonauta (Riot 
Cinema Collective \& Alcalá Alcalá, 2013 son dos ejemplos y modelos de vinculación de las audiencias al escenario audiovisual.

En Colombia también se han llevado a cabo experiencias de este tipo, siendo Cuentos de viejos (Piaggiodematei et al., 2012) una de las más representativas, tanto por la cantidad de personas vinculadas como por su permanencia en el tiempo. Si bien los referentes más claros se pueden encontrar en la industria cinematográfica o en los entornos digitales debido a las ventajas que aporta el internet, esto no significa que en medios tradicionales, como la televisión, este tipo de proyectos no hayan comenzado su curso. Uno de los formatos que siempre han buscado un relacionamiento directo con las audiencias es el informativo, y actualmente, con las nuevas tecnologías digitales, ha tratado de vincular a los televidentes a la construcción de sus contenidos.

Las diferentes formas de partición, a partir de sus particularidades o potencialidades, han comenzado a adquirir nombres. Ejemplo de esto es el término cocreación (proveniente de la expresión "creación colaborativa"), que se ha planteado como una creación en la que hay total reconocimiento y aporte de todos los actores involucrados (Quero \& Ventura, 2014). Ramón Salaverría, en entrevista con Adriana Vega, propone que los procesos de cocreación y participación entre los medios de comunicación y las personas se constituyen en una de las innovaciones del periodismo actual, al reevaluar la forma como se produce la información. Salaverría afirma que "cualquier medio que esté dando la espalda al público en el proceso de creación del relato periodístico es porque no entiende cuál es la dinámica del trabajo periodístico ahora" (Vega, 2019, p. 87).

Estas transformaciones que estamos viviendo para el proceso de producción de información y contenidos noticiosos motivaron este análisis. En él se abordaron las secciones "El periodista soy yo" (en adelante, EPSY) de Noticias Caracol (Caracol Televisión, 2016) y "El cazanoticias" (en adelante, EC) de Noticias RCN (RCN Televisión, 2016), a partir de tres ejes: la pieza audiovisual transmitida, los aportes del televidente y los aportes del noticiero, con el fin de establecer cómo son las dinámicas de relacionamiento de estos noticieros con sus televidentes y cómo funcionan los procesos de realización de estas secciones, para así determinar si la pieza audiovisual resultante corresponde a un proceso de cocreación o de participación según la definición que se ha construido para este análisis.

La pregunta que guía este análisis es, entonces, la que sigue: ¿son las secciones "El periodista soy yo" de Noticias Caracol y "El cazanoticias" de Noticias RCN procesos de cocreación audiovisual o corresponden a un proceso participativo? 


\section{Fundamento teórico}

Para dar respuesta a este interrogante, se ha construido una definición sobre cocreación, debido a la falta de una descripción clara y concisa en la literatura producida en los últimos años. Otros conceptos que se han considerado importantes y que deben ser abordados para la construcción de esta definición son transmedia y prosumidor, ya que dentro de cada uno de estos también se abordan las audiencias activas y las relaciones que se establecen entre estas y los medios de comunicación, al igual que las características que adquieren tales relaciones (Castells, 2009; Costa, 2013; Fernández, 2014; Islas-Carmona, 2008; Jenkins, 2003).

El estudio de los procesos de transformación que ha venido atravesando los procesos de producción y consumo de contenidos en la actualidad han comenzado a ser analizados desde campos concretos, como el periodismo. Renó y Flores (2018) definen el periodismo transmedia como aquella forma de lenguaje periodístico que contempla, simultáneamente, distintos medios, al igual que varios lenguajes y narrativas que circulan entre aquellos medios y que son pensados para una gran diversidad de públicos (2018, p. 53).

Dentro de la definición que estos autores proponen para el periodismo transmedia resaltan la importancia del teléfono móvil, ya que consideran que juega un papel fundamental en la construcción de estas narrativas por parte de las personas, al permitirles la movilidad y la instantaneidad en estos procesos de construcción colectiva. La definición propuesta por Renó y Flores nos permite conocer las características de los relatos denominados transmediales y por qué se consideran procesos participativos, aportando así información clave para nuestro entendimiento de los conceptos de cocreación y participación.

El concepto prosumidor se convirtió en un elemento clave dentro de esta investigación, por cuanto a partir de su definición pudimos comprender las características que adquiere un ciudadano o televidente cuando se vincula a los procesos de producción de contenidos o, incluso, cuando los inician. Scolari (2018), partiendo de la definición que Toffler acuñó en 1980, define al prosumidor como un creador participativo, que crea contenido nuevo y lo comparte en entornos digitales como las redes sociales. Scolari plantea que el prosumidor, para el ejercicio de sus actividades, desarrolla diferentes tipos de capacidades y conocimientos que le permiten crear y difundir su mensaje. Dentro de estas capacidades, señala el autor, se encuentra la gestión de redes sociales y entornos digitales, el uso de dispositivos electrónicos, como cámaras, y el uso de software especializado para los procesos de posproducción o para la programación de los entornos digitales. 
Estas competencias las desarrolla el prosumidor junto con las de distribución del contenido, la remezcla y aquellas para la gestión de espacios participativos. Esta definición del concepto prosumidor nos permitió comprender las cualidades que debíamos analizarles a los televidentes que enviaban sus notas a los informativos, con el fin de determinar si su accionar correspondía con el de un prosumidor.

Respecto al concepto cocreación, este ha sido abordado desde diferentes disciplinas, como el mercadeo, la administración y la educación, al igual que en las industrias creativas. Uno de los investigadores que lo ha hecho es John Banks, quien establece en entrevista con Jenkins que "la cocreación se refiere a la práctica a través de la cual usuarios y consumidores tienen un papel activo en la generación de valor para la producción y el consumo cultural. Así, los aportes que realice un consumidor son considerados de gran valor" (Jenkins, 2014, p.19).

Vladimir Zwass (2010) define la cocreación como la participación de consumidores junto con productores en la creación de valor en el mercado. Este investigador plantea que la definición reduccionista del consumidor, los diversos papeles que una persona desempeña y que afectan al mercado y las motivaciones que van más allá de lo monetario son argumentos que demuestran la importancia de las investigaciones sobre el tema. Zwass, respecto a los procesos de cocreación, resalta que van más allá de la simple personalización de un producto, pues se producen mayormente en espacios virtuales, permitiendo relaciones entre los participantes de la actividad. Este proceso puede ser iniciado tanto por las empresas como por los consumidores, para satisfacer las necesidades de individuos concretos (Zwass, 2010). Estas relaciones, señala finalmente, pueden estar impregnadas de sentimientos de pertenencia que se producen alrededor de los procesos creativos, al igual que pueden proveer identidad a sus participantes.

Prahalad y Ramaswamy (2004) proponen una metodología de cocreación que apunta a la creación de valor agregado en productos a partir de la relación de las empresas con clientes y comunidades de interés. Las posibilidades de conexión de los usuarios hacen viable la interacción de estos no solo con las empresas, sino también con comunidades especializadas. Con el propósito de generar una participación y desarrollar cocreaciones efectivas, Prahalad y Ramaswamy proponen la metodología DART, sustentada en cinco principios: el diálogo, el diagnóstico, el acceso, el riesgo y la transparencia (Prahalad \& Ramaswamy, 2004).

Por otra parte, Johnson y Johnson (1999) proponen una caracterización de los procesos de aprendizaje colaborativo que contribuye a establecer las etapas y entornos en los que se desarrolla la cocreación. Estos investigadores 
plantean la "interacción promotora" como un estímulo y la consolidación de esfuerzos para alcanzar un objetivo, realizar tareas y producir en pro de las metas de un grupo. Y señalan la importancia de analizar los diversos procesos comunicativos que se dan entre todos los sujetos participantes de los trabajos colaborativos (Johnson \& Johnson, 1999).

A partir de los postulados de Banks (Jenkins, 2014), Zwass (2010), Prahalad y Ramaswamy (2004), y Johnson y Johnson (1999), se construyó la definición de cocreación que guía a este análisis. Se entiende por cocreación, entonces, un proceso de interacción comunicativa que busca dar respuesta o mejorar un problema o situación; los aportes que realizan los participantes pueden llegar a tener el mismo valor, aunque no se materialicen de la misma forma.

La cocreación es intencionada y estimulada por un proponente claro, que es quien coordina todo el proceso. No hay limitación ni exclusión de las formas de participación: las limitaciones radican exclusivamente en las capacidades de los participantes para responder a la situación planteada. Finalmente, es una actividad que se puede dar de forma asincrónica, pues los entornos digitales se convierten en un espacio que dota de nuevos atributos a este proceso.

\section{Metodología}

Para este análisis se seleccionaron 10 emisiones de cada una de las secciones (EPSY y EC), emitidas durante abril de 2016 en la segunda emisión del día de cada uno de los noticieros. Estas secciones fueron seleccionadas debido a que, dentro del imaginario de académicos y no académicos, son un referente claro al hablar de cocreación. En la actualidad, estas secciones forman parte de las estrategias de participación que ambos noticieros han mantenido de manera constante por más de nueve años. A estas razones se suma el discurso de los presentadores, en el que invitan a los televidentes a participar, lo que muestra la intención de creación conjunta.

Otro de los motivos para seleccionar estas secciones fue su similitud, pues son formatos audiovisuales transmitidos en canales de televisión nacional privados, lo que potencializa la posibilidad de participación de los televidentes de diferentes regiones de Colombia. Así, se podría presentar una mayor variedad de matices en las formas de vinculación de los usuarios con los medios y en la tipología misma de los televidentes participantes. En un primer momento, durante la selección de la muestra, se realizó una primera revisión del material que se analizaría para constatar que compartiera estas características mínimas. Luego de identificados estos mínimos entre las secciones, se seleccionó y obtuvo la muestra. 
Para el análisis del material audiovisual se diseñó una tabla de clasificación, constituida por tres ejes fundamentales: la pieza audiovisual transmitida, los aportes del televidente y los aportes del noticiero a dicha construcción; a partir de estos se evaluó la existencia de ciertas características en cada pieza. Las variables establecidas tenían como fin identificar el nivel de participación e injerencia, tanto del medio como del televidente, en la construcción de las notas periodísticas y en el desarrollo de cada una de estas. Cada eje estaba constituido por diferentes variables, como muestra la Tabla.

\section{Tabla. Ejes y variables de análisis}

\begin{tabular}{|c|c|c|}
\hline \multicolumn{3}{|c|}{ Matriz de análisis de notas periodísticas } \\
\hline Eje 1. Pieza audiovisual & Eje 2. Televidente & Eje 3. Noticiero \\
\hline Variables & Variables & Variables \\
\hline $\begin{array}{l}\text { Código de la pieza } \\
\text { audiovisual. }\end{array}$ & Sexo del televidente. & $\begin{array}{l}\text { Se brindan indicaciones } \\
\text { al televidente sobre cómo } \\
\text { participar. }\end{array}$ \\
\hline $\begin{array}{l}\text { Sección a la que pertenecía } \\
\text { el contenido. }\end{array}$ & $\begin{array}{l}\text { Aparición del televidente } \\
\text { en la nota. }\end{array}$ & $\begin{array}{l}\text { Se realiza contraste y } \\
\text { verificación con las entidades } \\
\text { respectivas. }\end{array}$ \\
\hline Duración de la sección. & $\begin{array}{l}\text { El televidente asume } \\
\text { el papel de reportero } \\
\text { presentador. }\end{array}$ & $\begin{array}{l}\text { El noticiero adquiere } \\
\text { algún compromiso con el } \\
\text { televidente. }\end{array}$ \\
\hline $\begin{array}{l}\text { Cantidad de notas por } \\
\text { sección. }\end{array}$ & $\begin{array}{l}\text { El televidente manifiesta } \\
\text { expresamente su interés. }\end{array}$ & \\
\hline Sinopsis de la nota. & $\begin{array}{l}\text { Entidad denunciada o } \\
\text { acusada. }\end{array}$ & \\
\hline Duración de la nota. & $\begin{array}{l}\text { El televidente gestiona } \\
\text { testimonios. }\end{array}$ & \\
\hline $\begin{array}{l}\text { Ubicación de la nota en la } \\
\text { sección. }\end{array}$ & $\begin{array}{l}\text { El televidente representa } \\
\text { explícitamente a una } \\
\text { comunidad. }\end{array}$ & \\
\hline \multicolumn{3}{|l|}{$\begin{array}{l}\text { Existencia de propuesta } \\
\text { narrativa. }\end{array}$} \\
\hline \multicolumn{3}{|l|}{$\begin{array}{l}\text { Temática del contenido } \\
\text { audiovisual. }\end{array}$} \\
\hline \multicolumn{3}{|l|}{$\begin{array}{l}\text { Cumple con criterios de } \\
\text { calidad de la información. }\end{array}$} \\
\hline $\begin{array}{l}\text { Cumple las indicaciones } \\
\text { técnicas dadas por el } \\
\text { noticiero. }\end{array}$ & & \\
\hline
\end{tabular}

Fuente: Elaboración propia. 


\section{Eje 1. Pieza audiovisual}

\section{Variables}

- Código de la pieza audiovisual. Código único de identificación de cada pieza de video.

- Sección a la que pertenecía el contenido.

- Duración de la sección. Total de segundos de duración de toda la sección.

- Cantidad de notas por sección. Las secciones EPSY y EC están compuestas por diversas piezas enviadas por los televidentes. Se buscaba identificar cuántas piezas componían cada una de las emisiones de las secciones.

- Sinopsis de la nota. Una descripción del contenido de la nota. Se construyó un enunciado corto en el que se diera respuesta a quién habla, qué dice, dónde se encuentra y cuál es la situación planteada.

- Duración de la nota. Total de segundos de duración de cada una de las notas. Dentro de esta medición fueron tenidos en cuenta los pronunciamientos que el presentador del noticiero hacía antes o después de la nota.

- Ubicación de la nota en la sección. Este dato se estableció a partir del total de la sección.

- Existencia de una propuesta narrativa. Se buscaba establecer si había una propuesta narrativa de parte del televidente. Se cumplía con este parámetro si dentro de la nota emitida se identificaba el uso de diferentes planos audiovisuales por parte del noticiero o de los televidentes, con el fin de dar una mayor descripción y contextualización de los sucesos que planteaban en su relato.

- Temática del contenido audiovisual. Se buscaba identificar las temáticas más recurrentes dentro de los videos enviados por los televidentes. Partiendo de la previsualización de los contenidos, se propusieron las siguientes categorías: obras de infraestructura pública, educación, infracciones de tránsito y otras.

- Cumple con criterios de calidad de la información. Se buscaba establecer la calidad de la información de la pieza a través de estadísticas, cifras, contextualización, relación de antecedentes, pluralidad de voces y relación de las entidades o personas involucradas.

- Cumple las indicaciones técnicas dadas por el noticiero. Teniendo en cuenta la previsualización de las piezas, en las que se evidenció que los noticieros brindan unas indicaciones precisas, se buscaba establecer si dichas pautas eran acatadas por los televidentes en la construcción de las notas. 


\section{Eje 2. Televidente}

\section{Variables}

- Sexo del televidente. Se buscaba establecer el sexo del televidente que enviaba las piezas audiovisuales al noticiero.

- Aparición del televidente en la nota. Se buscaba establecer el tipo de presencia que el televidente tenía dentro de la pieza audiovisual. Esta presencia debía ser explícita y materializada en la cámara o con su voz.

- El televidente asume el papel de reportero presentador. Se buscaba establecer el nivel de aparición y el papel del televidente dentro de la pieza, con el fin de identificar si se dirigía a la cámara y conducía el relato como lo haría un reportero.

- El televidente manifiesta expresamente su interés. Se busca establecer la motivación del televidente para la realización de la pieza audiovisual. con base en la previsualización del material, se plantearon como opciones de respuesta: denuncia, queja o resaltar acciones positivas.

- Entidad denunciada o acusada. Se buscaba establecer contra qué institución u organización iba dirigida la denuncia. Después de la previsualización, se establecieron como categorías: Estado, empresa privada, particular.

- El televidente gestiona testimonios. Se buscaba establecer si dentro de la construcción de la pieza audiovisual se presentaban testimonios de diferentes personas o entidades con el fin de detallar las particularidades de la situación.

- El televidente representa explícitamente a una comunidad. Se buscaba establecer si el televidente vinculaba de forma explícita a una comunidad (como vocero).

\section{Eje 3. Noticiero}

\section{Variables}

- Se brindan indicaciones al televidente sobre cómo participar. Partiendo de la previsualización del material, se supo que el noticiero daba indicaciones específicas sobre cómo construir el material audiovisual.

- Se realiza contraste y verificación con las entidades respectivas. Se buscaba establecer si el noticiero gestionaba entrevistas o declaraciones con personas o entidades que ayudaran a comprender la globalidad y los diferentes puntos de vista de la situación.

- El noticiero adquiere algún compromiso con el televidente. Se buscaba establecer si había algún interés del noticiero en darle continuidad o acompañamiento a la solicitud que el televidente manifestaba en la 
nota transmitida; esto se debía evidenciar mediante algún compromiso explícito.

Las piezas audiovisuales que conformaron la muestra fueron descargadas de los portales web de los noticieros, con el fin de realizar las cuatro visualizaciones necesarias para la identificación de las características incluidas en la tabla. En EPSY, se identificaron varias notas en una sola emisión: cada una fue analizada de manera individual, pero entendida dentro del contexto de la sección. Así, el total de unidades de análisis fue 36 notas, agrupadas en 20 emisiones.

Este análisis contempló una metodología mixta, la cual partía de los datos cuantitativos obtenidos en cada una de las variables ya descritas. Los datos cuantitativos obtenidos fueron cruzados e interrelacionados para identificar las particularidades de las secciones de ambos noticieros, con especial interés en el proceso de construcción de la nota que se emitió por televisión. Una vez analizadas las notas periodísticas, se establecieron criterios que debían cumplir para ser entendidas como procesos de cocreación. Estos criterios fueron fijados a partir de la definición que se construyó alrededor de los aportes de varios autores citados en esta investigación:

- Interés expreso del televidente dentro de la pieza audiovisual.

- La nota emitida debe observar los principios periodísticos retomados en esta investigación (Gutiérrez Coba, 2006).

- Debe haber un aporte a la construcción de la nota por parte del noticiero y del televidente. Ambos deben aportar, indiferente del porcentaje en que lo hagan, contraste o verificación con las instancias implicadas en el pronunciamiento del televidente, cifras, antecedentes y demás datos contextuales que ayuden a comprender el suceso.

- Uno de los actores presentes en el proceso debe coordinar las actividades de construcción de la nota.

\section{Resultados}

Aunque a partir de las variables que se analizaron en cada una de las notas periodísticas y del cruce entre ellas se puede obtener gran cantidad de datos, para responder la pregunta que orientó esta investigación se retomaron aquellas variables que, de manera individual o en contraste con otras, brindaran información que permitiera determinar el grado de participación que el televidente y el noticiero tuvieron en la construcción del material audiovisual transmitido. 
En la variable que indagaba sobre la aparición del televidente (a través de su imagen o su voz) en la pieza audiovisual, se obtuvo que en el 100 \% de las notas de EC el televidente aparece. En EPSY, el televidente tuvo algún tipo de participación en el 75 \% de las notas y en el 25 \% restante solo contribuyó a la construcción del material como un proveedor de imágenes, sin participación creativa.

En EC, en el 100 \% de las notas el noticiero gestionó fuentes que sirvieron para verificación o contraste frente a lo planteado por el televidente, mientras que en EPSY esta gestión únicamente se dio en el 4 \%.

En la definición de cocreación planteada se resalta la importancia de que uno de los actores coordine el proceso creativo. Esta característica, por la naturaleza propia del medio y las dinámicas de producción y emisión, recae sobre el noticiero. En EC no se presentó ninguna indicación concreta sobre cómo los televidentes debían participar. En EPSY las indicaciones estuvieron presentes en el $58 \%$ del material analizado.

Estos datos muestran que los noticieros tienen el dominio del proceso de producción, pues en el 100 \% de las notas de EC se pudo determinar que la producción estuvo a cargo de Noticias RCN, por el uso de micrófonos con distintivos corporativos y la uniformidad de los elementos gráficos añadidos en posproducción. Este nivel de participación del equipo de producción no se dio en el caso de EPSY. Las indicaciones brindadas por Noticias Caracol fueron claras y reiterativas para garantizar unos mínimos de calidad en las imágenes que los televidentes enviaban, pues su equipo participaba solamente de la etapa de edición y posproducción.

Otro aspecto importante que se identificó es la relación entre la duración de la nota, las posibilidades de participación y el cumplimiento de los criterios de calidad informativa. EC es una sección compuesta por una sola nota, lo que permite que la nota creada adquiera ciertas cualidades: una propuesta narrativa, que el televidente aparezca en la nota y que se cumplan los criterios de calidad informativa en el 100 \% de los contenidos emitidos. EPSY está compuesta, generalmente, por tres notas, lo que se relaciona con el hecho de que el noticiero no domine los procesos de producción. Por esto, del contenido que envían los televidentes, deben escoger las mejores tomas audiovisuales, lo que se materializa en notas con una duración menor respecto a las emitidas en la sección EC.

La ubicación de la nota dentro de la sección de Noticias Caracol corresponde al cumplimiento de los criterios de calidad técnica de la imagen y a la posibilidad de aparición del televidente en ella. Así, las que se ubican en el primer lugar de la sección son aquellas que cumplen con estas dos 
variables, mientras las ubicadas en el tercer lugar pertenecen al $85 \%$ que no cumple con criterios de calidad de la imagen y no cuenta con la aparición del televidente.

Los aportes realizados (o la ausencia de aportes) por cada uno de los noticieros a la construcción de las notas se convierten en un elemento importante para conocer el nivel de apertura hacia los televidentes que desean ser parte de los procesos de producción. Como EC domina todo el proceso de producción, el aporte del televidente se ve limitado a plantear la situación o dar su testimonio. En EPSY, el televidente provee el material audiovisual y en algunos casos también cifras, datos o pronunciamientos de otros televidentes para contextualizar el tema, lo que muestra una participación clara en la construcción de las notas. Estos aportes de los televidentes a EPSY no se ven recompensados con la participación del noticiero, pues como informativo se limitaron a transmitir las notas sin agregar elementos que las enriquecieran en términos informativos.

El dominio de los noticieros sobre el proceso de producción se convierte en un limitante de la libertad de cocreación de los ciudadanos. En EC, el pronunciamiento del televidente se debe adaptar a la propuesta narrativa y estética que el noticiero ha construido para la sección, y aquella es replicada en cada una de las notas, sin importar el tema o la población. En el caso de EPSY, en las notas que se ubican en el tercer lugar, la participación del televidente es aún más limitada, pues la única presencia de aquel sería su voz fuera de cámara relatando los sucesos que registra con su celular, pero esta es suprimida por el noticiero y remplazada por la locución del presentador de la sección. Así, el televidente vuelve a quedar relegado a proveedor de imágenes y no es un participante de la construcción de los contenidos periodísticos.

El análisis de la muestra deja claro que las actividades que se deben llevar a cabo para la construcción de una nota periodística con calidad informativa pueden ser desarrolladas, de igual forma, por los televidentes y por los dos noticieros. Lo único que no puede hacer el televidente, y que es el fundamento de estas secciones, es la transmisión de los contenidos en un medio masivo de comunicación. Realidad esta que, en la actualidad y gracias al internet y a la evolución del consumidor a prosumidor, está cambiando. Sibien hoy los televidentestienen más instrumentos, mediosy conocimientos, que les permiten ser prosumidores, es importante reconocer el papel e importancia de los medios y de los profesionales de la información, no solo por el dominio de las herramientas de transmisión, sino por el conocimiento especializado en la construcción de relatos periodísticos, como lo señalan Rodríguez, Hermoso y Calvo (2007), quienes abogan por una estructura de 
producción de noticias e información en la que se reconozca el potencial de cada uno de los actores involucrados.

Por su parte, la transformación en prosumidor se puede evidenciar en algunas de las notas analizadas dentro de la sección EPSY: en esta, el cumplimiento de las indicaciones del noticiero se dio en el 50 \% del contenido. Entre tanto, la gestión de fuentes y los procesos de posproducción que los mismos televidentes realizaron en algunas de estas notas son muestra de cómo las audiencias están comenzando a dominar procesos de producción audiovisual y de la capacidad de construcción de narraciones audiovisuales que han ido adquiriendo.

Con base en los hallazgos identificados en el análisis de los datos registrados en la tabla de sistematización y ciertos patrones de correspondencia entre algunas variables, y a la luz de los aspectos seleccionados para determinar si las notas emitidas eran cocreaciones o no, concluimos que dos de las 10 notas analizadas en EC corresponden a cocreación y que ninguna de las 26 de EPSY cumplió con los criterios establecidos en este análisis.

\section{Conclusiones}

En Colombia hay diferentes elementos que permitirían desarrollar procesos de cocreación entre los medios y sus audiencias: infraestructuras tecnológicas suficientes, una ciudadanía activa, canales de comunicación de alcance nacional y, principalmente, temas sobre los cuales los actores sociales se quieren pronunciar. A pesar de este escenario, ninguna de las dos secciones informativas analizadas llega a desarrollar una propuesta de cocreación sólida con los televidentes.

Los canales de televisión aún deben trabajar en el reconocimiento de los televidentes como actores activos dentro de la industria audiovisual, pues ello es uno de los elementos más importantes en la actualidad para la gestión de marcas periodísticas, como lo señalan Burguera Pérez \& VaraMiguel (2018) y Hartmann (2015) al platear la necesidad de reestructurar la forma en que se gestionan tales marcas, por cuanto ya no se pueden pensar solo como activos estratégicos para explotar las posibilidades comerciales de un mercado y conseguir beneficios, sino como plataformas sociales y culturales en las que tienen lugar procesos de cocreación.

Secciones como EC y EPSY se convierten, entonces, en estrategias de los noticieros para incluir a los televidentes en sus contenidos, pero bajo los procedimientos por ellos establecidos y no bajo procesos de mutuo desarrollo en los que ambos actores tengan las mismas posibilidades y 
libertades. Los intereses de los medios por generar estos espacios podrían estar relacionados con la idea de crear en los televidentes la sensación de cobertura nacional con su despliegue periodístico al trasmitir videos enviados desde regiones remotas. También podrían estar relacionados con la búsqueda de aceptación por parte de los televidentes al apoyar sus denuncias y, finalmente, con la intención de abaratar costos de producción.

El televidente es vinculado al proceso de creación de estas notas como un "proponente de temas", como el detonante de una situación sobre la cual el noticiero pone su interés o, simplemente, como un proveedor de contenidos. La participación del televidente se limita a proponer el tema y a ser fuente directa del hecho. Las limitaciones no se presentan solo del lado del televidente: en EPSY no aparece lo que podríamos denominar una autolimitación de funciones por parte del medio, pues este no gestionó el $92 \%$ del contenido analizado (algún elemento complementario como entrevistas, testimonios o imágenes que contribuyeran a darle valor informativo al contenido desarrollado inicialmente por el televidente). Su función, en la mayoría de los casos analizados, se limitó a la transmisión de la nota construida por el televidente. Este hallazgo es corroborado con el obtenido por Pachón Mayorga (2019) en un análisis realizado sobre esta misma sección del informativo de Noticias Caracol. La autora concluye:

Esta sección de "El periodista soy yo" de Noticias Caracol procura poner en práctica el seguimiento a cada uno de los contenidos que llegan al medio, sin embargo, este se queda corto debido a que la catalogación, administración y contestación por parte de los entes gubernamentales hacia el medio de comunicación es rezagada (Pachón Mayorga, 2019, p. 199).

Esta conclusión señala que la falta de seguimiento a las noticias y de una construcción narrativa del suceso es fruto, a su vez, de la falta de una pronta respuesta de los entes gubernamentales, principales denunciados por los ciudadanos. En todo caso, queda claro que el papel del informativo se limita a la transmisión del contenido.

Desarrollar procesos de participación efectivos, en los cuales se reconozca el potencial de las audiencias, es uno de los retos actuales de los noticieros colombianos. Las limitaciones impuestas a los televidentes en estas experiencias pueden ser un punto importante para comprender la proliferación de sitios web, perfiles en redes sociales y contenidos en diversas plataformas digitales cuya temática principal son las denuncias ciudadanas.

Por lo anterior, ha comenzado la migración de las audiencias televisivas al internet por las posibilidades que presenta este entorno: entre muchas 
otras, una comunicación horizontal, multidireccional y de muchos a muchos, además de la posibilidad de conformar comunidades y espacios para una creación participativa que reconozca la voz de todos.

\section{Referencias}

Burguera Pérez, M. \& Vara-Miguel, A. (2018). Branding e integración de las audiencias en la creación de la marca periodística. Miguel Hernández Communication Journal, 9 (2), 491509. http://dx.doi.org/10.21134/mhcj.v0i9.261

Caracol Televisión (Productor) (2016). El periodista soy yo [Noticiero de televisión]. http:// www.caracoltv.com/

Castells, M. (2009). Comunicación y poder. Alianza Editorial.

Costa, C. (2013). Narrativas transmedia nativas: ventajas, elementos de la planificación de un proyecto audiovisual transmedia y estudio de caso. Historia y Comunicación Social, (18), 561-574. http:/ / revistas.ucm.es/index.php/HICS/article/view/ 44349

Fernández, P. (2014). Consumos culturales en América Latina y la emergencia del prosumidor: un recorrido conceptual desde la sociedad de la información. Revista Communication Papers, 4 (3), 87-100. https://dialnet.unirioja.es/servlet/ articulo? codigo $=4785939$

Gutiérrez Coba, L. (2006). Análisis de la calidad informativa, primer paso hacia el cambio. Palabra Clave, 9 (1), 29-56. http: / / www.redalyc.org/articulo.oa?id=64900102

Hartmann, B. J. (2015). Media brand cultures: researching and theorizing how consumers engage in the social construction of media brands. En G. Siegert, K. Förster, S. M. ChanOlmsted \& M. Ots. (Edits.), Handbook of media branding (pp. 217-229). Springer, Cham.

Islas-Carmona, J. (2008). El prosumidor. El actor comunicativo de la sociedad de la ubicuidad. Palabra Clave, 11 (1), 29-39. http: / / palabraclave.unisabana.edu.co/index.php/ palabraclave/article/view/1413

Jenkins, H. (2003). Transmedia storytelling. Technology Review. Revista del Massachusetts Institute of Technology (MIT), 4 (1).

Jenkins, H. (8 de septiembre de 2014). Transmedia 202: reflexiones adicionales [Blog]. http:/ / henryjenkins.org/2014/09/transmedia-202-reflexionesadicionales.html\#sthash. tSv6aETP.dpuf

Johnson, D. \& Johnson, R. (1999). Aprender juntos y solos. Aprendizaje cooperativo, competitivo e individualista. Aique.

Pachón Mayorga, M. (2019). La apropiación de las TIC (dispositivos móviles) por parte de los ciudadanos colombianos y el aprovechamiento mediático de sus contenidos. Caso: El periodista soy yo de Noticias Caracol (Tesis de maestría, Universidad Autónoma de Occidente, Cali, Colombia).

Piaggiodematei, Señal Colombia, Hierro (productores), Dematei, M., Ferrer, A., Piaggio, L \& Smith, C (Directores) (2012). Cuentos de viejos [Proyecto transmedia]. Piaggiodematei, Señal Colombia y Hierro Productora.

Prahalad, C. \& Ramaswamy, V. (2004). El futuro de la competencia: creación conjunta de valor único con los consumidores. Ediciones Gestión 2000. 
Quero, M. \& Ventura, R. (2014). Análisis de las relaciones de Co-creación de valor. Un estudio de casos de crowdfunding. Universia Business, 1 (1), 128-143. https:/ / ubr. universia.net/article/viewFile/914/1044

RCN Televisión (Productor) (2016). El cazanoticias [Noticiero de televisión]. http://www. canalrcn.com/

Renó, D. \& Flores, J. (2018). Periodismo transmedia. Ria Editorial.

Riot Cinema Collective (Productores) \& Alcalá, N. (Director) (2013). El Cosmonauta [Cinta cinematográfica]. Riot Cinema.

Rodríguez, E. R., Hermoso, S. P. \& Calvo, P. A. (2007). Periodismo ciudadano versus periodismo profesional: ¿somos todos periodistas? Estudios sobre el Mensaje Periodístico, 13, 189-212.

Scolari, C. A. (2018). Introducción. En C. A. Scolari (Edit.), Del alfabetismo mediático al alfabetismo transmedia. Adolescentes, medios de comunicación y culturas colaborativas. Aprovechando las competencias transmedia de los jóvenes en el aula (pp.14-23). Transliteracy - H2020 Research and Innovation Actions.

Scott, R. (Productor) \& Macdonald, K. (Director) (2011). Life in a day [Cinta cinematográfica]. YouTube - Scott Free Productions.

Toffler, A. (1981). La tercera ola. Edivisión.

Vega, A. M. (2019). El big data y los desafíos innovadores para el periodismo. Comunicación, (39), 79-89.

Zwass, V. (2010). Co-Creation: toward a taxonomy and an integrated research perspective. International Journal of Electronic Commerce, 1 (15), 11-48. 10.2753/JEC1086-4415150101 